\title{
Anomalous Tunneling of Phonon Excitations between Two Bose-Einstein Condensates
}

\author{
Yu. Kagan, D. L. Kovrizhin, and L. A. Maksimov \\ "RRC Kurchatov Institute," Kurchatov Square 1, 123182 Moscow, Russia
}

(Received 15 October 2002; published 2 April 2003)

\begin{abstract}
We discuss the tunneling of phonon excitations across a potential barrier separating two condensates. It is shown that a strong barrier proves to be transparent for the excitations at low energy $\varepsilon$. Moreover, the transmission is reduced with increasing $\varepsilon$ in contrast to the standard dependence. This anomalous behavior is due to the existence of a quasiresonance interaction. The origin of this interaction is a result of the formation of a special well determined by the density distribution of the condensate in the vicinity of a high barrier.
\end{abstract}

DOI: $10.1103 /$ PhysRevLett.90.130402

The investigation of tunneling phenomena in BoseEinstein condensed systems becomes one of the most evolving trends in the Bose-Einstein condensation studies of ultracold atomic gases (see, e.g., [1] and references therein). The possibility to create a potential barrier for atoms using resonant laser light as a sheet or by using it as an optical lattice led to numerous experimental studies. The great advantage of this method is due to the fact that barrier forming in this way does not destroy the condensate, and one can change the barrier by simply changing the intensity of light. One of the interesting aspects in this field is associated with the problem of tunneling of collective excitations possessing specific features in the condensed phase. A condensate moving at velocity $v$ smaller than some critical velocity $v_{c}$ transmits across a potential barrier without scattering and reflection. Excitations of a condensate at rest, in particular, sound excitations, should experience the tunneling transmission together with the reflection. It is significant that the motion of excitations takes place at the background of the inhomogeneous built-up distribution of the condensate density $g(x)$. The inevitable reduction of $g(x)$ near the barrier results in the appearance of a potential well. For a sufficiently high barrier $V \gg \mu$, where $\mu$ is the chemical potential, the tunneling of excitations, as we will see below, demonstrates an anomalous character. On the one hand, the barrier proves to be transparent for phonon excitations within a limited range of low energies. On the other hand, the coefficient of tunneling transmission $T$ reduces as the energy of excitations $\varepsilon$ grows. This is in contrast to the typical growth of $T$ with $\varepsilon$. Such anomalous behavior is associated with the appearance of a quasibound state in the continuous spectrum. The formation of the quasibound state is connected with the specific behavior of function $g(x)$ in the vicinity of the barrier. Provided the size $L$ of the condensate is much larger than the correlation length $\xi=\hbar(m \mu)^{-1 / 2}$ and the barrier is high, the dependence $g$ on $x$ shows a universal character. As a result the anomalous tunneling shows universal character as well.

To get results in a more straightforward way, we consider a one-dimensional symmetrical configuration
PACS numbers: 03.75.Lm, 05.30.Jp

formed by two identical condensates separated by a rectangular barrier with a height $V_{0} \gg \mu$. The general equation for the Heisenberg field operator of particles $\hat{\Psi}(x, t)$ reads

$i \hbar \frac{\partial \hat{\Psi}}{\partial t}=\left(-\frac{\hbar^{2}}{2 m} \frac{\partial^{2}}{\partial x^{2}}-\mu\right) \hat{\Psi}+V(x) \hat{\Psi}+U \hat{\Psi}^{+} \hat{\Psi} \hat{\Psi}$.

Hereafter it is implicitly assumed that the transverse size of the system is sufficiently large. Accordingly, a collision of atoms has a three-dimensional character and, as usual, we take for the interaction $U=4 \pi \hbar^{2} a_{0} / m$, where $a_{0}$ is the scattering length. We consider only the case $a_{0}>0$.

We start from the consideration of the ground state assuming temperature $T=0$. In this case, the operator $\hat{\Psi}$ can be replaced by the macroscopic condensate wave function $\Psi(x)$. The equation for $\Psi(x)$ in the dimensionless form can be written as

$$
i \frac{\partial \bar{\Psi}}{\partial \bar{t}}=\left(-\frac{1}{2} \frac{\partial^{2}}{\partial \bar{x}^{2}}-1\right) \bar{\Psi}+\bar{V}(\bar{x}) \bar{\Psi}+|\bar{\Psi}|^{2} \bar{\Psi} .
$$

Here we introduced the following notations:

$$
\begin{aligned}
& \bar{x}=x / \xi, \quad \bar{t}=\mu t / \hbar, \quad \bar{\Psi}=\Psi(U / \mu)^{1 / 2}, \\
& \bar{V}=V / \mu=\left(V_{0} / \mu\right) \theta(a-|x|) .
\end{aligned}
$$

Further on we shall omit the bar for all variables in Eq. (2).

Let us find a stationary solution $\Psi_{0}(x)$ of Eq. (2), assuming that $\Psi_{0}(x)=1$ for $|x| \gg 1$. Outside barrier Eq. (2) has the well-known static solution

$$
\Psi_{0}(x)=\tanh \left(|x|+x_{0}\right) .
$$

Under the barrier, we can find the first integral of Eq. (2), taking into account the condition $\Psi_{0}^{\prime}(0)=0$,

$$
\Psi_{0}^{\prime}(x)=\sqrt{\left(\Psi_{0}^{2}-b^{2}\right)\left(\Psi_{0}^{2}+\kappa_{0}^{2}\right)} .
$$

Here $b^{2}=\Psi^{2}(0), \kappa_{0}^{2}=2\left(V_{0}-1\right)+b^{2}$. The general solution takes the form

$$
\Psi_{0}(x)=\frac{b}{c n\left(\sqrt{b^{2}+\kappa_{0}^{2}} x, q\right)}, \quad q=\frac{\kappa_{0}}{\sqrt{b^{2}+\kappa_{0}^{2}}},
$$


where $c n(u, q)$ is the Jacobi elliptic function (see, e.g., [2]).

In the case of a strong barrier when $\kappa_{0} \gg 1$ and $\kappa_{0} d>1(d=2 a$ is the barrier width), one can neglect the nonlinearity related to the interparticle interaction. Equating solutions (4) and (6) and their derivatives at the boundary of the barrier $|x|=a$, we derive

$$
\Psi_{0}(x) \cong\left(\kappa_{0} \sinh \kappa_{0} a\right)^{-1} \cosh \kappa_{0} x, \quad \Psi_{0}(a) \approx \kappa_{0}^{-1} \ll 1 .
$$

Let us now consider the tunneling of excitations propagating at the background of the distribution of the condensate density. These excitations can be found as oscillations of the classical field of a condensate (see, e.g., [3]). For this purpose, let us represent the function $\Psi$ in Eq. (2) as $\Psi=\Psi_{0}+\Psi^{\prime}$ and linearize the equation in $\Psi^{\prime}$,

$$
\begin{gathered}
i \frac{\partial \Psi^{\prime}}{\partial t}=\hat{h} \Psi^{\prime}+g(x) \Psi^{\prime *}, \\
\hat{h}=-\frac{1}{2} \frac{d^{2}}{d x^{2}}+V(x)+2 g(x)-1, \quad g(x)=\Psi_{0}^{2}(x) .
\end{gathered}
$$

For excitations with energy $\varepsilon$, we will seek the solution of a set of Eqs. (8) for $\Psi^{\prime}$ and $\Psi^{\prime *}$ in the form [3],

$$
\Psi^{\prime}=u(x) e^{-i \varepsilon t}-v^{*}(x) e^{i \varepsilon t} .
$$

Then we have

$$
(\hat{h}-\varepsilon) u-g v=0, \quad-g u+(\hat{h}+\varepsilon) v=0 .
$$

Introducing the notations

$$
S=u+v, \quad G=u-v,
$$

and combining these equations, we arrive at a fourthorder differential equation,

$$
(\hat{h}+g)(\hat{h}-g) S=\varepsilon^{2} S .
$$

If the solution of $S(x)$ is known, one can readily find the function $G(x)$,

$$
\varepsilon G=(\hat{h}-g) S .
$$

Far from the barrier at $|x| \gg 1$, we have $g(x) \rightarrow 1$. The solutions of Eq. (12) in this uniform region are sought as $S \sim \exp (i k x)$. As a result, we find four roots,

$$
\begin{aligned}
& k_{1,2}= \pm k, \quad k=\sqrt{2}\left(\sqrt{1+\varepsilon^{2}}-1\right)^{1 / 2}, \\
& k_{3,4}=\mp i q, \quad q=\sqrt{2}\left(\sqrt{1+\varepsilon^{2}}+1\right)^{1 / 2} .
\end{aligned}
$$

The first two roots correspond to the Bogoliubov spectrum (in the dimensional variables $k \rightarrow k \xi$ and $\varepsilon \rightarrow \varepsilon / \mu$, respectively). The solutions corresponding to $k_{3,4}$ do not contribute to the asymptote of the general solution at $|x| \gg 1$ but prove to be significant in the regions close to the barrier. For $\varepsilon \ll 1$, we have

$$
k=\varepsilon, \quad q=2,
$$

neglecting terms of order $\varepsilon^{2}$.

Outside the barrier the general solution can be represented as a linear superposition of the solutions $S_{n}(x)$ of
Eq. (12) corresponding to the roots given in (14). Let the excitations come from the left-hand side. Then, omitting the divergent components, we have

$$
\begin{array}{ll}
S(x)=A S_{1}(x)+B S_{2}(x)+C S_{3}(x), & x<-a, \\
S(x)=D S_{1}(x)+F S_{4}(x), & x>a .
\end{array}
$$

Here the functions $S_{n}(x)$ for fixed $\varepsilon$ have a universal form

$$
S_{n}(x)=e^{i k_{n} x}\left[\tanh \left(|x|+x_{0}\right)-\frac{i k_{n}}{2} \operatorname{sgn} x\right] .
$$

$G(x)$ can be found from (13) by conserving the same set of coefficients to be determined as in (16).

Consider now the underbarrier region. Since $g(x) \leq$ $\kappa_{0}^{-2} \ll 1$, we first find the solution of Eq. (12) omitting $g(x)$. In this case Eq. (12) can be rewritten as

$$
\left(-\frac{1}{2} \frac{d^{2}}{d x^{2}}+V-1\right)^{2} S=\varepsilon^{2} S .
$$

Inserting the solution as $S \sim \exp (-\kappa x)$ into the equation, we again find four roots

$\kappa_{1,2}= \pm \kappa_{+}, \quad \kappa_{3,4}= \pm \kappa_{-}, \quad \kappa_{ \pm}=\kappa_{0} \sqrt{1 \pm 2 \varepsilon / \kappa_{0}^{2}}$.

Accordingly, the general solution for the underbarrier region reads

$$
S_{B}=K e^{\kappa_{+} x}+L e^{-\kappa_{+} x}+M e^{\kappa_{-} x}+N e^{-\kappa_{-} x} .
$$

Substituting this expression into (13) together with (19), we arrive at

$$
G_{B}=-\left(K e^{\kappa_{+} x}+L e^{-\kappa_{+} x}\right)+M e^{\kappa_{-} x}+N e^{-\kappa_{-} x} .
$$

Now, employing $\varepsilon / \kappa_{0}^{2} \ll 1$ and $\varepsilon a / \kappa_{0} \ll 1 \quad$ [in dimensional variables $\varepsilon / V_{0} \ll 1$ and $\left(\varepsilon / V_{0}\right) \kappa_{0} a \ll 1$ correspondingly] we can replace $\kappa_{ \pm}$by $\kappa_{0}$. As a result, we have

$$
\begin{aligned}
S_{B} & =(M+K) e^{\kappa_{0} x}+(N+L) e^{-\kappa_{0} x}, \\
G_{B} & =(M-K) e^{\kappa_{0} x}+(N-L) e^{-\kappa_{0} x} .
\end{aligned}
$$

The boundary conditions at $x= \pm a$ yield eight equations for the determination of all coefficients in (16) and (20)

$$
\begin{aligned}
& S( \pm a)=S_{B}( \pm a)(\alpha), \quad G( \pm a)=G_{B}( \pm a)(\beta), \\
& \left.\frac{d S}{d x}\right|_{ \pm a}=\left.\frac{d S_{B}}{d x}\right|_{ \pm a}(\gamma),\left.\quad \frac{d G}{d x}\right|_{ \pm a}=\left.\frac{d G_{B}}{d x}\right|_{ \pm a}(\delta) .
\end{aligned}
$$

In order to find the solution in the explicit form, we focus entirely on the tunneling and reflection of collective excitations in the sound region, corresponding to the roots (15). In addition, we simplify a set of equations by omitting quadratic terms such as $k^{2}, k \zeta^{1}, \zeta^{2}(\zeta=$ $\left.\kappa_{0}^{-1} \ll 1\right)$, and making use of the smallness of the coefficients $C$ and $F$ of the order of $(k, \zeta)$ with respect to the other coefficients in Eq. (16). This simplification yields 
the equalities $M \approx K$ and $N \approx L$ resulting from Eq. $[(21) \delta]$. Together with the boundary conditions for $G$ $[(21) \beta]$ this gives

$$
\bar{F}=-\bar{D} \frac{i k}{2}, \quad \bar{C}=\bar{A} \frac{i k}{2}-\bar{B} \frac{i k}{2},
$$

with

$$
\begin{array}{ll}
\bar{F}=F e^{-2 a}, & \bar{C}=C e^{-2 a}, \quad \bar{B}=B e^{i k a}, \\
\bar{D}=D e^{i k a}, & \bar{A}=A e^{-i k a} .
\end{array}
$$

The remaining set of four equations can be solved straightforwardly:

$$
\begin{aligned}
\bar{B} & =\frac{\bar{A}}{Z}\left[-k^{2}+\left(k^{2}+4 \zeta^{2}\right) e^{-4 \kappa_{0} a}\right], \\
\bar{D} & =\frac{\bar{A}}{Z} 4 i k \zeta e^{-2 \kappa_{0} a}, \quad K=\frac{\bar{A}}{Z} \zeta k(k+2 i \zeta) e^{-3 \kappa_{0} a}, \\
L & =\frac{\bar{A}}{Z} \zeta k^{2} e^{-\kappa_{0} a}, \quad Z=k^{2}-(k+2 i \zeta)^{2} e^{-4 \kappa_{0} a} .
\end{aligned}
$$

As a result, we find for the tunneling transmission of the excitations in terms of the dimensional variables,

$$
T=\left|\frac{D}{A}\right|^{2} \approx \frac{16\left(k / \kappa_{0}\right)^{2} e^{-4 \kappa_{0} a}}{\left\{(k \xi)^{2}+\left[4\left(\kappa_{0} \xi\right)^{-2}-(k \xi)^{2}\right] e^{-4 \kappa_{0} a}\right\}^{2}} .
$$

Even for a wide barrier, the transmission coefficient demonstrates practically the full transparency at

$$
k=k_{*} \approx \frac{1}{\xi} \frac{2}{\kappa_{0} \xi} e^{-2 \kappa_{0} a} .
$$

For $k \gg k_{*}$, but keeping $k \xi \ll 1$, the transmission coefficient falls with increasing $k$ :

$$
T \approx \frac{16 e^{-4 \kappa_{0} a}}{\left(\kappa_{0} \xi\right)^{2}(k \xi)^{2}} .
$$

This result is in clear contrast to the typical enhancement of $T$ with increasing $k$.

Let us now consider a more accurate solution for the underbarrier region, involving the condensate density $g(x)$ in Eqs. (12) and (13). The explicit form for $g(x)$ can be obtained from the results (6) and (7) for $\Psi_{0}(x)$. Numerical calculation must be performed in order to find the solution of Eqs. (12) and (13) for this region. The general solution implies again the use of the boundary condition of (21). The results for the dependence $T$ on $k$ are presented in Fig. 1. In these figures, the function $T(k)$, obtained by ignoring $g(x)$ in the underbarrier region, is shown by dashed lines. The exact solution shows again the existence of the full transparency. The only difference is a shift of the maximum of $T$ approaching $k=0$. With increasing $k$, in particular, in the region where the asymptotic expression (25) is correct, both curves coincide. We intentionally have chosen quite different sets of parameters in Figs. 1(a) and 1(b) in order to demonstrate the
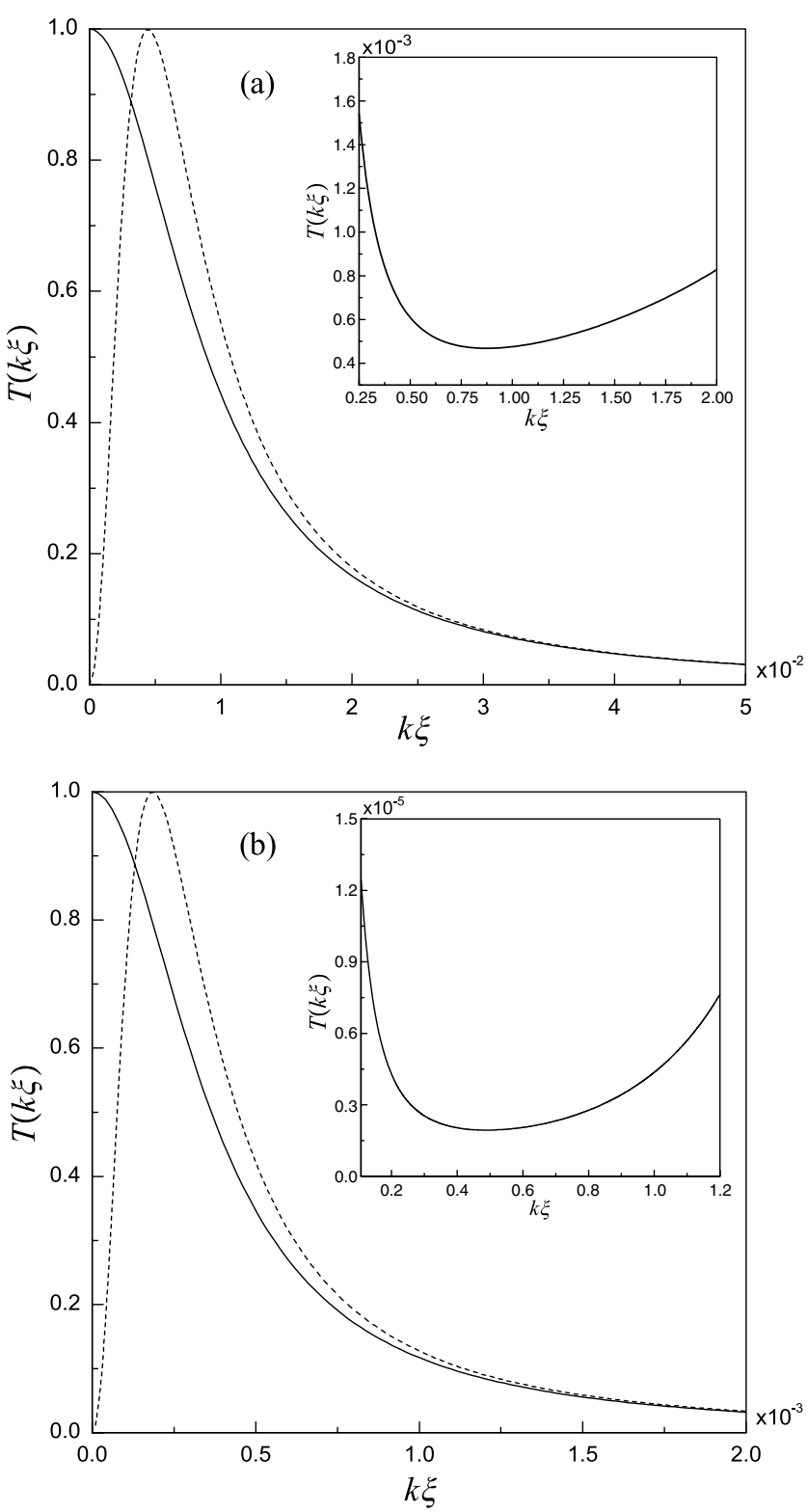

FIG. 1. The coefficient of tunneling transmission $T$, as a function of $k \xi$ for two sets of parameters: (a) $\kappa_{0} \xi=8$, $\kappa_{0} d=4$; (b) $\kappa_{0} \xi=3, \kappa_{0} d=8$. Dashed line corresponds to $T(k \xi)$ obtained with neglecting $g(x)$ in the underbarrier region.

rather universal character of the $T(k)$ dependence. In fact, shown only is the change of the scale of the $k$ region where the transparency is high.

The anomalous behavior of the tunneling transmission of excitations has an interesting origin. In the vicinity of the barrier, the condensate density decreases as $\tanh ^{2}\left[\left(|x|+x_{0}\right) / \xi\right]$, producing potential wells for the excitations on both sides of the barrier. In the case of a strong barrier, the condition $\left(|x|+x_{0}\right) / \xi \ll 1$ holds. In this case, a virtual resonance level in the continuous spectrum close to $\varepsilon=0$ appears. This results in the drastic change of the tunneling picture. As $k$ increases, 
the quasiresonance scattering decreases, entailing the unusual reduction of $T(k)$. At $k \xi \sim 1$, the role of the virtual level reduces and the normal growth of $T$ with $k$ should be restored. We can see it from the insets of Figs. 1(a) and 1(b), where the dependence $T(k)$ is plotted just for this interval of $k$. Note that in all cases the anomalous tunneling can be revealed considering the reflection coefficient,

$$
R=|B / A|^{2}=1-T .
$$

Treating the tunneling of phonon excitations, it is interesting to trace the formation of the energy flux $Q$. This flux can be found employing the local form of the energy conservation law. Provided the Hamiltonian is represented as

$$
\begin{aligned}
H & =\int E(r) d^{3} r, \\
E(r) & =\frac{\hbar^{2}}{2 m} \nabla \hat{\Psi}^{+} \nabla \hat{\Psi}+\hat{\Psi}^{+}(V-\mu) \hat{\Psi}+\frac{U}{2} \hat{\Psi}^{+} \hat{\Psi}^{+} \hat{\Psi} \hat{\Psi},
\end{aligned}
$$

then

$$
\frac{\partial E}{\partial t}=-\operatorname{div} Q .
$$

Using Eqs. (27) and (1), we find

$$
Q=-\frac{\hbar^{2}}{m} \operatorname{Re}\left(\frac{\partial \hat{\Psi}^{+}}{\partial t} \frac{\partial \hat{\Psi}}{\partial x}\right) .
$$

Assuming as before that the condensate is at rest, we consider the energy transfer by excitations. Treating them as oscillations of the classic field of a condensate, we have the following from (29) for the flux averaged over time:

$$
\langle Q\rangle=n_{0} \frac{\mu^{3 / 2}}{m^{1 / 2}}\langle\bar{Q}\rangle, \quad\langle\bar{Q}\rangle=-\operatorname{Re}\left\langle\frac{\partial \Psi^{\prime *}}{\partial t} \frac{\partial \Psi^{\prime}}{\partial x}\right\rangle .
$$

Here $n_{0}$ is the total particle density, $\bar{Q}$ is the expression for the energy flux defined in terms of dimensionless units (3). Let us consider again the incident flux of excitations with energy $\varepsilon$. Inserting the function $\Psi^{\prime}$ from (10) and using (11), we obtain

$$
\langle\bar{Q}\rangle=\frac{\varepsilon}{2} \operatorname{Im}\left(S^{*} \frac{d S}{d x}+G^{*} \frac{d G}{d x}\right) .
$$

Outside the barrier, we use the general solution for $S$ in the form (16) and (17). Taking into account that the function $S_{3}$ is real and $S_{2}(k, x)=S_{1}^{*}(k, x)=S_{1}(-k, x)$, we find for the region $x<-a$,

$$
\operatorname{Im}\left(S^{*} \frac{d S}{d x}\right)=|A|^{2}(1-R) \frac{k}{2}\left[1+\tanh ^{2}\left(x-x_{0}\right)\right],
$$

where $R$ is the reflection coefficient (26). According to
(13), the contribution from the function $G$ to (31) equals

$$
\operatorname{Im}\left(G^{*} \frac{d G}{d x}\right)=|A|^{2}(1-R) \frac{k}{2}\left[1-\tanh ^{2}\left(x-x_{0}\right)\right] .
$$

The sum of both expressions is independent of $x$, demonstrating the conservation of the energy flux of excitations.

Let us find the relation between $|A|^{2}$ and the physical parameters. Comparing the density of the noncondensate particles $n^{\prime}=(1 / 2) n_{0}|A|^{2}$ in the incident phonon flux with the known Bogoliubov result (see, e.g., [4]) for the relation between $n^{\prime}$ and phonon density $n(k)$ concentrated in the $k$-mode $n^{\prime}=\left(\mu / \hbar c_{0} k\right) n(k)$, we find

$$
|A|^{2} \approx 2\left(\mu / \hbar c_{0} k\right)\left[n(k) / n_{0}\right] .
$$

Here $c_{0}=\sqrt{\mu / m}$ is the speed of sound defined for the density at $|x| \gg a$.

Returning to the dimensional units, we find for the energy flux (30)

$$
\langle Q\rangle=n(k) c_{0} \varepsilon(1-R) .
$$

In order to obtain the flux under the potential barrier in the explicit form, we neglect again the distribution of the condensate density in this region. Taking into account the solution (20) and the conditions $M \approx K$ and $N \approx L$, we find from (31) $\langle\bar{Q}\rangle=4 \varepsilon \kappa_{0} \operatorname{Im}\left(K L^{*}\right)$. The coefficients $K$ and $L$ are determined in (23) with $|A|^{2}$ according to (32). As a result, the energy flux (30) acquires the value

$$
\langle Q\rangle=n(k) c_{0} \varepsilon T,
$$

where $T$ is the transmission coefficient (24). At $x \geq a$, the flux has the same value (34). Expressions (33) and (34) demonstrate the constancy of the energy flux in the system [see Eq. (26)]. Note that this is not the case for the particle flux. One can show that this flux, which is inevitably connected with the transfer of phonon excitations, is not conserved and depends on $x$.

Let us note that the obtained results are not just an outcome of the rectangular barrier. Actually, all results hold good for a smooth potential barrier of arbitrary form, in particular, for that created by resonance laser light.

This work is supported by INTAS (Project No. 20012344), by Netherlands Organization for Science Research (NWO), and by the Russian Foundation for Basic Research.

[1] A. J. Leggett, Rev. Mod. Phys. 73, 307 (2001).

[2] I.S. Gradshteyn and I. M. Ryzhik, Table of Integrals, Series, and Products (Academic, London, 1994), 5th ed.

[3] F. Dalfovo, S. Giorgini, L. P. Pitaevskii, and S. Stringari, Rev. Mod. Phys. 71, 463 (1999).

[4] E. M. Lifshitz and L. P. Pitaevskii, Statistical Mechanics (Pergamon, Oxford, 1988), Pt. 2. 